\title{
3D-SOP Millimeter-Wave Functions For High Data Rate Wireless Systems Using LTCC and LCP technologies
}

\author{
J.-H.Lee, S.Sarkar, S.Pinel, J.Papapolymerou, J.Laskar and M.M.Tentzeris \\ Georgia Electronic Design Center, School of Electrical and Computer Engineering \\ Georgia Institute of Technology, Atlanta GA 30332-0269 USA \\ Fax:(404) 894-0222, Email:jonglee@ece.gatech.edu
}

\begin{abstract}
In this paper, the development of three-dimensional (3-D) millimeter-wave functions in multilayer low temperature cofired ceramic (LTCC) and liquid crystal polymer (LCP) technologies is presented for millimeter-wave compact and easy-to-design passive solutions for high data rate wireless systems. Both ceramic and organic technologies are candidates for the 3-D integration of system-on-package (SOP) miniaturized RF/microwave/millimeter-wave systems. LTCC has been widely used as a packaging material because of its process maturity/stability and its relatively high dielectric constant that enables a significant reduction in the module/function dimensions. As an alternative, LCP is an organic material that offers a unique combination of electrical, chemical, and mechanical properties, enabling high-frequency designs due to its ability to act as both the substrate and the package for flexible and conformal multilayer functions. A LTCC patch resonator filter that uses vertical coupling overlap and transverse cuts as design parameters has been designed to achieve a high level of miniaturization and a great compromise between compactness and power handling. Excellent agreement between the simulation and the measurement has been verified for two operating frequency bands $(58-60 \mathrm{GHz} / 38-40 \mathrm{GHz})$ of RF communications and sensors for applications such as wireless broadband internet or inter-satellite communications. A band pass filter has been fabricated on LCP substrate, offering a very simple, low loss flexible and low lost filtering solution for wideband millimeter waves applications such as $60 \mathrm{GHz}$ WLAN short-range gigabit wireless systems. The design exploits the ripple near the cut off frequency of Tchebysheff low pass filter to create a band pass response and it exhibits the insertion loss as low as $1.5 \mathrm{~dB}$ at the center frequency of $60 \mathrm{GHz}$ and $3-\mathrm{dB}$ bandwidth of $16.7 \%(\sim 10 \mathrm{GHz})$.
\end{abstract}

\section{Introduction}

Millimeter-wave (mmW) electronics for commercial applications, such as short-range broadband wireless communications and automotive collision avoidance radars, require low-manufacturing cost, excellent performance, and high level of integration. The multilayer LTCC System-OnPackage (SOP) approach is very well suited for these requirements because it offers a great potential for passives' integration and enables microwave devices to be fabricated with high reliability, while maintaining the low cost [1]. The very mature multilayer fabrication capabilities of LTCC $\left(\varepsilon_{\mathrm{r}}=5.4, \tan \delta=0.0015\right)$ up to $100+\mathrm{GHz}$ enable the replacement of broadside coupling by vertical coupling and make LTCC a competitive solution to meet millimeter wave design requirements. As an alternative, Liquid Crystal Polymer (LCP) is an organic material that offers a unique low-cost all-in-one solution for high frequency designs due to its ability to act as both a high-performance flexible substrate $\left(\varepsilon_{\mathrm{r}}=2.9-3.0\right.$, $\tan \delta=0.002-0.004)$ and a near-hermetic package for multilayer modules [2-4]. These characteristics make LCP very appealing for many applications and it can be viewed as a prime technology for enabling system-on-package RF and millimeter-wave (mmW) designs [2]. The choice of the most suitable technology depends on the application specifications such as environment, operation frequency, performance, volume and cost.

In this paper, we present the development of various advanced 3-D LTCC and LCP system-on-package passive components enabling a complete passive solution for compact, low cost wireless front-end systems to be used in RF and $\mathrm{mmW}$ frequency ranges. The design of single-mode slotted patch filter with a transverse cut on each side has been presented to achieve compactness and great compromise between size and power handling. This filter has been embedded in LTCC and designed for two operating frequencies $(58-60 \mathrm{GHz} / 38-40 \mathrm{GHz})$ of mobile communication systems. The paper clearly demonstrates how the physical dimensions of the miniaturized filter are determined by showing the simulated filter response as a function of critical design parameters such as the length of transverse cuts and the overlap distance between feeding structure and patch resonator. Also, a band filter has been designed to enable a low cost, very easy to design filtering solution to be integrated into a wireless millimeter-wave gigabit link system. The ripple of modified stepped impedance Tchebysheff low pass filters has been exploited and combined with a rejection centered at $60 \mathrm{GHz}$. Insertion loss as low as $1.5 \mathrm{~dB}$ has been measured, demonstrating the potential of LCP for millimeter-wave applications.

\section{Patch Resonator Filter (59.3 GHz/39GHz) on LTCC}

Integrating filter on-package in LTCC multilayer technology is a very attractive option for RF front-ends up to mm-wave frequency range in terms of both miniaturization by vertical deployment of filter elements and reduction of the number of components and assembly cost by eliminating the demand for discrete filters. In mm-wave frequencies the band pass filters are commonly realized using slotted patch resonators due to their miniaturized size, their excellent compromise between size, power handling and easy-to-design layout [5]. In this section, the design of a single-pole slotted patch filter is presented for two operating frequency bands (38-40 GHz and 58-60GHz). All designs have been simulated 


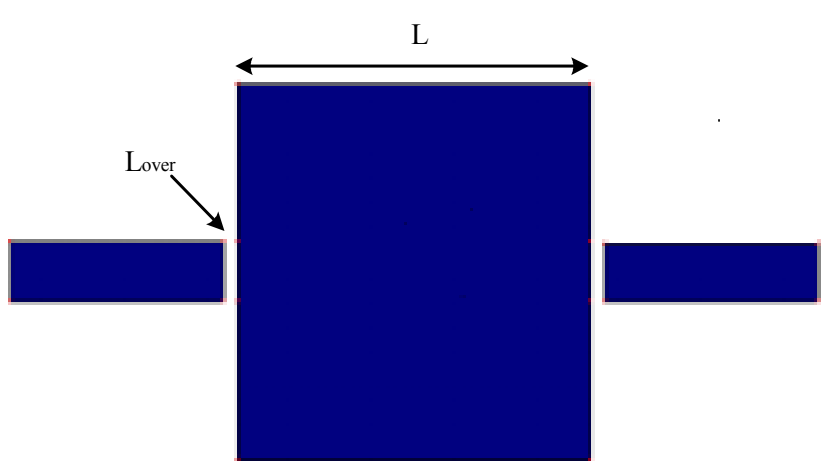

(a)

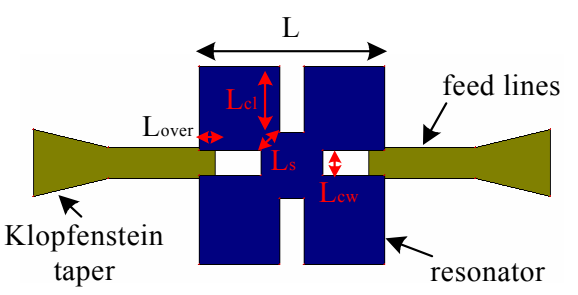

(b)

Fig. 1 Top view of (a) conventional $\lambda / 2$ square patch (b) Miniaturized patch resonator.

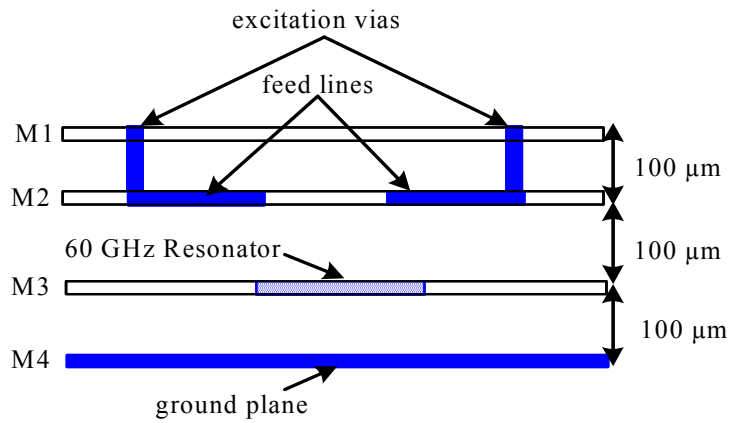

(a)

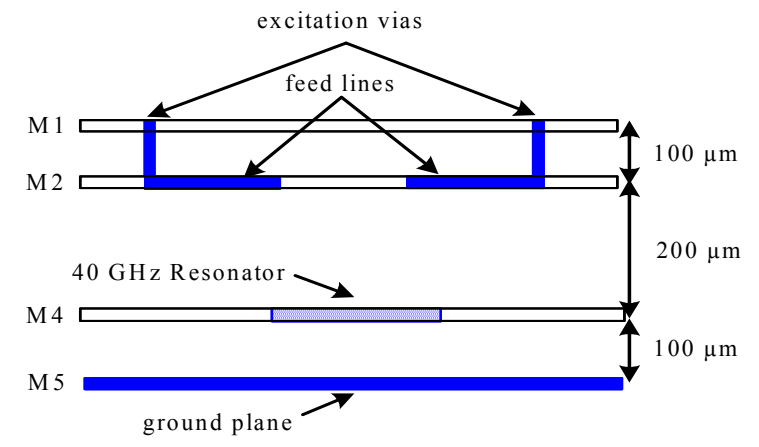

(b)

Fig. 2 Side view of (a) $60 \mathrm{GHz}$ slotted patch resonator (b) $39 \mathrm{GHz}$ slotted patch resonator

using the MOM-based, 2.5D full-wave solver IE3D. Fig. 1 shows a top-view comparison between basic half-wavelength square patch resonator $(\mathrm{L} \times \mathrm{L}=0.996 \mathrm{~mm} \times 0.996 \mathrm{~mm})[5]$ (Fig. 1 (a)) and the new configuration $(\mathrm{L} \times \mathrm{L}=0.616 \mathrm{~mm} \times$ $0.616 \mathrm{~mm})$ capable to provide good tradeoffs between miniaturization and power handling (Fig. 1 (b)). Side view and the photograph of the resonators for two operating frequency bands are shown in Fig. 2 and Fig. 3, respectively. In the design of $\lambda / 2$ square patch, the planar single-mode patch and microstrip feedlines are located at metal 3 (M3 in Fig. 2 (a))

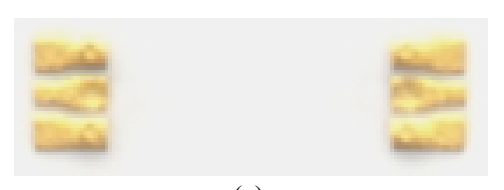

(a)

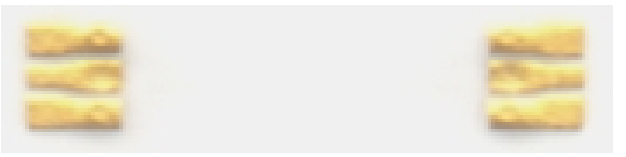

(b)

Fig. 3. The photographs of fabricated filters with coplanar waveguide (CPW) pads (a) at $60 \mathrm{GHz}$ (b) at $39 \mathrm{GHz}$

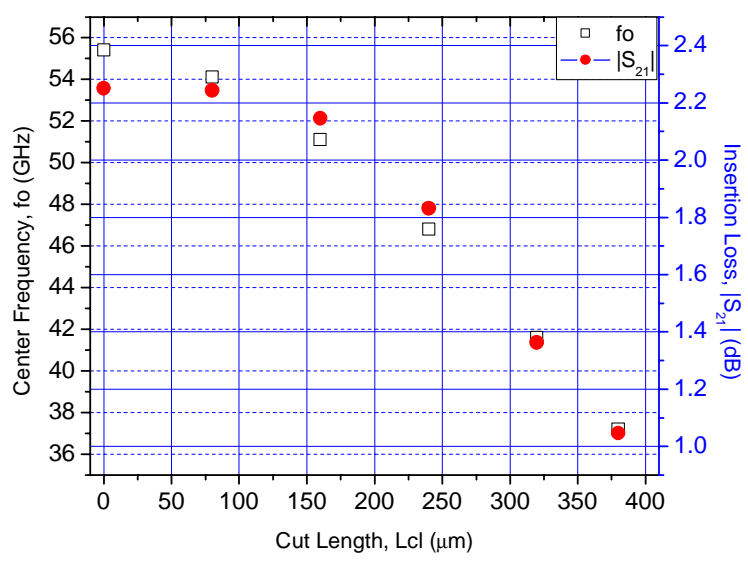

Fig. 4 Simulated responses of center frequency $\left(f_{0}\right)$ and insertion loss $\left(\left|S_{21}\right|\right)$ as a function of transverse cut $\left(\mathrm{L}_{\mathrm{cl}}\right)$

and use the end-gap capacitive coupling between the feedlines and the resonator itself in order to achieve $3 \% 3 \mathrm{~dB}$ bandwidth and $\angle 3 \mathrm{~dB}$ insertion loss around the center frequency of 60 GHz. However, the required coupling capacitances to obtain design specifications could not be achieved because of the LTCC design rules limitations. In order to maximize the coupling strength, while minimizing the effects of the fabrication, the proposed novel structure takes advantage of the vertical deployment of filter elements by placing the feed lines and the resonator into different vertical metal layers as shown as Fig. 2 (a). This transition also introduces a 7.6\% frequency downshift because of the additional capacitive coupling effect as compared to the basic $\lambda / 2$ square patch resonator (Fig. 1 (a)) directly attached by feedlines.

Transverse cuts have been added on each side of the patch in order to achieve significant miniaturization of the patch by adding additional inductance. Fig. 4 shows the simulated response for the center frequency and the insertion loss as the length of cuts $\left(\mathrm{L}_{\mathrm{cl}}\right.$ in Fig. 1 (b)) increases while the fixed width of cuts $\left(\mathrm{L}_{\mathrm{cw}}=\mathrm{L} / 8\right.$ in Fig. 1 (b)) is determined by the fabrication tolerance. It can be observed that the operating frequency range shifts further downward about $33 \%$ as the length of cut $\left(\mathrm{L}_{\mathrm{cl}}\right.$ in Fig. 1 (b)) increases by approximately $379 \mu \mathrm{m}$. Additional miniaturization is limited by the minimum distance (Ls in Fig. 1 (b)) between the corners of adjacent orthogonal cuts. Meanwhile, as the operating frequency decreases, the shunt conductance in the equivalent circuit of the single patch also decreases because its value is reciprocal to the 


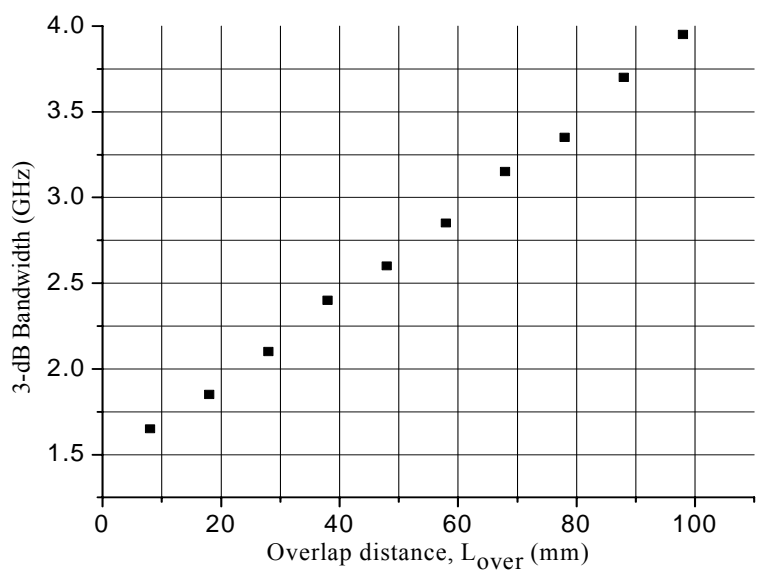

Fig. 5 Simulated 3-dB bandwidth as function of Overlap distance of (a) 60 $\mathrm{GHz}$ slotted patch resonator (b) $39 \mathrm{GHz}$ slotted patch resonator

exponential function of the operating frequency [6]. This fact additionally causes the reduction of radiation loss since it is proportionally related to the conductance in the absence of conductor loss [7]. Therefore, insertion loss at resonance is improved from $2.27 \mathrm{~dB}$ to $1.06 \mathrm{~dB}$ by an increase of $\mathrm{L}_{\mathrm{cl}}$ in Fig. 1 (b).

The patch size is reduced significantly from $0.996 \mathrm{~mm}$ to $0.616 \mathrm{~mm}$. The modification of bandwidth due to the patch's miniaturization can be compensated by adjusting the overlap distance $\left(\mathrm{L}_{\text {over }}\right)$. Fig.5 shows the simulated response for 3-dB bandwidth as $\mathrm{L}_{\text {over }}$ increases. It is observed that $3-\mathrm{dB}$ bandwidth increases as $\mathrm{L}_{\text {over }}$ increases due to a stronger coupling effect and then $\mathrm{L}_{\text {over }}$ is determined to be $18 \mu \mathrm{m}$ corresponding to $1.85 \mathrm{GHz} 3-\mathrm{dB}$ bandwidth.

The proposed embedded microstrip line filters are excited through vias connecting the coplanar waveguide signal pads on the top metal layer (M1 in Fig. 2 (a)), reducing radiation loss compared to microstrip lines on the top (surface) layer. As shown in Fig. 1 (b), Klopfenstein impedance tapers are used to connect the 50 ? feeding line and the via pad on the metal 2 (M2 in Fig. 1 b)). The overlap $\left(\mathrm{L}_{\text {over }}{ }^{2} \mathrm{~L} / 31\right)$ and transverse cuts $\left(\mathrm{L}_{\mathrm{cw}}{ }^{-} \mathrm{L} / 8, \quad \mathrm{~L}_{\mathrm{cl}}{ }^{2} \mathrm{~L} / 3.26\right)$ have been finally determined to achieve desired filter characteristics with aid of IE3D. Fig. 3 (a) shows the photograph of the fabricated filter prototypes with coplanar waveguide (CPW) pads fabricated in LTCC $\left(\varepsilon_{\mathrm{r}}=5.4, \tan \delta=0.0015\right)$ with a dielectric layer thickness of $100 \mu \mathrm{m}$ and metal thickness of $9 \mu \mathrm{m}$. The overall size is $4.018 \mathrm{~mm} \times 1.140 \mathrm{~mm} \times 0.3 \mathrm{~mm}$ including the CPW measurement pads. As shown in Fig. 6 (a), the experimental and the simulated results agree very well. It can be easily observed that the insertion loss is $<2.3 \mathrm{~dB}$, the return loss $>25.3 \mathrm{~dB}$ over the pass band and the $3 \mathrm{~dB}$ bandwidth is about $1 \mathrm{GHz}$. The center frequency shift from $59.85 \mathrm{GHz}$ to 59.3 $\mathrm{GHz}$ can be attributed to the fabrication accuracy (vertical coupling overlap affected by the alignment between layers, layer thickness tolerance). This was the first fabrication iteration and the differences could be corrected in the second and third iterations.

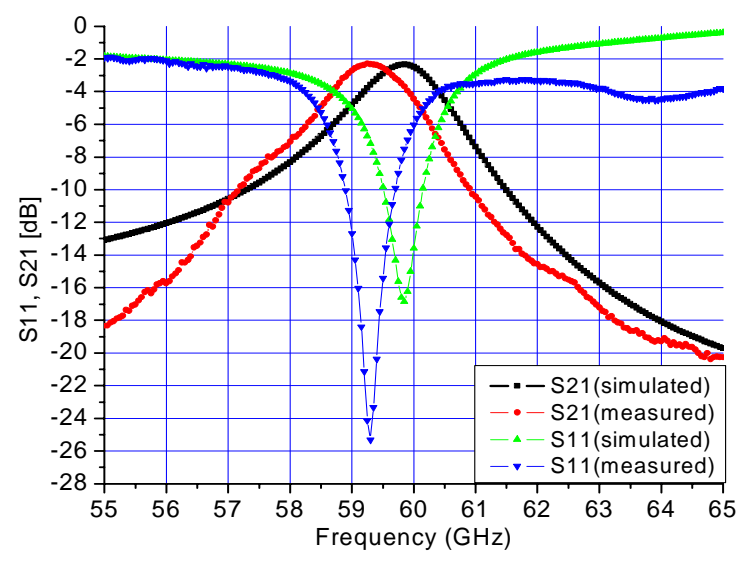

(a)

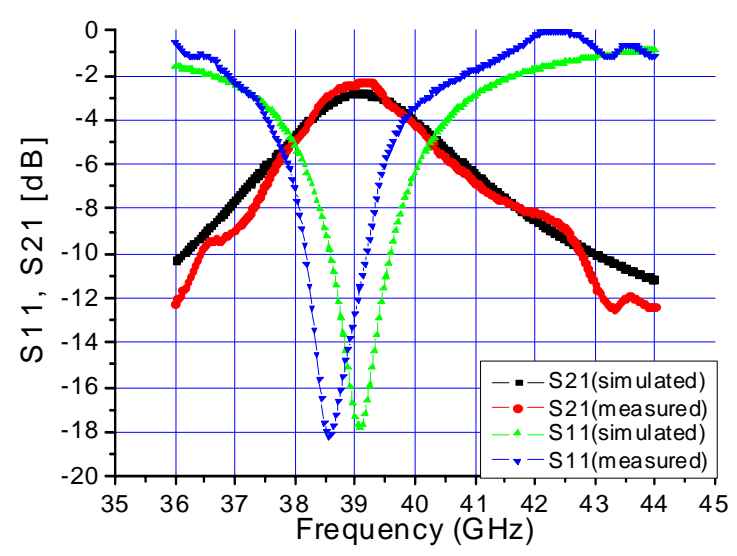

(b)

Fig. 6 Measured and simulated S-parameters of (a) $60 \mathrm{GHz}$ slotted patch resonator (b) $39 \mathrm{GHz}$ slotted patch resonator

The proposed slotted-patch filter architecture was also used in the design of mobile satellite communication systems around $39 \mathrm{GHz}$. The $39 \mathrm{GHz}$ single-mode patch filter $(\mathrm{L} \times \mathrm{L}=1.021 \mathrm{~mm} \times 1.021 \mathrm{~mm})$ was designed for $3.08 \%$ bandwidth, $39 \mathrm{GHz}$ center frequency, and $<3 \mathrm{~dB}$ insertion loss. The design procedure was similar to the $60 \mathrm{GHz}$ filter except from the fact that the resonator was placed on two layers (instead of one layer) beneath the feeding lines as shown in the side view of Fig. 2 (b). The overlap $\left(\mathrm{L}_{\text {over }}{ }^{2} \mathrm{~L} / 7\right)$ and the transverse cuts $\left(\mathrm{L}_{\mathrm{cw}}{ }^{-} \mathrm{L} / 8.2, \mathrm{~L}_{\mathrm{cl}}{ }^{\sim} \mathrm{L} / 3\right)$ were determined to achieve the desired filter characteristics. The overall size was $4.423 \mathrm{~mm} \times 1.140 \mathrm{~mm} \times 0.4 \mathrm{~mm}$ with $\mathrm{CPW}$ measurement pads. The fabricated filter (Fig. 3 (b)) exhibits a minimum insertion loss of $2.3 \mathrm{~dB}$, a return loss of $18.2 \mathrm{~dB}$, and a $3-\mathrm{dB}$ bandwidth about 3\%. (Fig. 6 (b)) The experimental error in the calculation of the center frequency can be attributed to the fabrication accuracy as mentioned in $60 \mathrm{GHz}$ case.

\section{Millimeter-Wave Compact Band Pass Filter (BPF) Design on LCP}

We developed a $60 \mathrm{GHz}$ band pass filter with excellent performance on LCP. The device manipulates the ripple near the cut off frequency a Tchebyscheff low pass filter to create a 


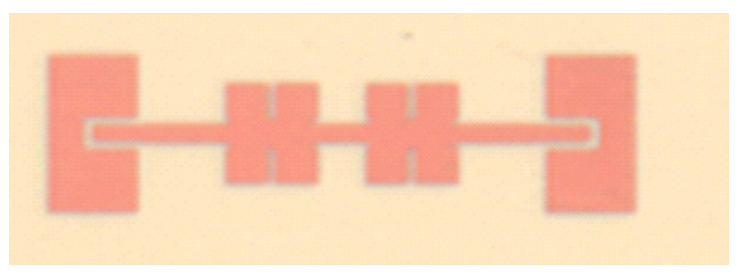

(a)

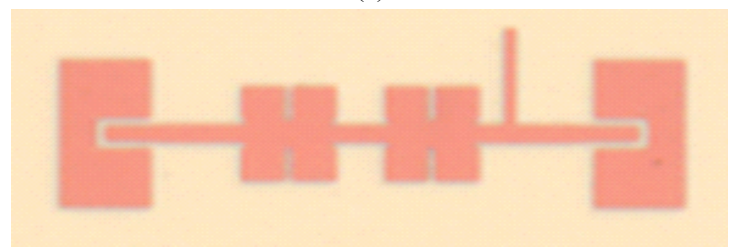

(b)

Fig. 7 Photograph of the fabricated millimetre-wave LCP band pass filter with (a) two resonators (b) two resonators and a rejection stub

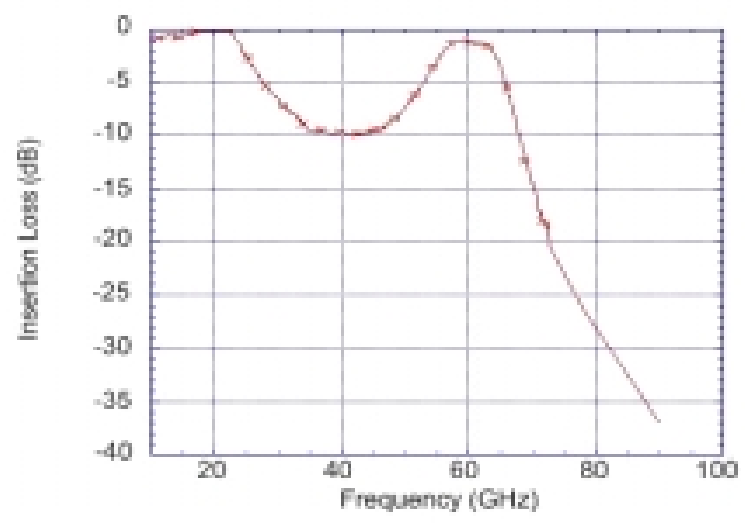

(a)

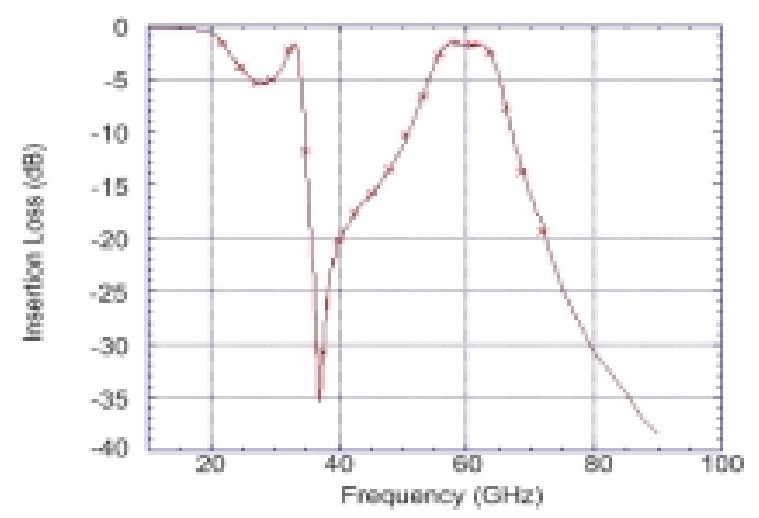

(b)

Fig. 8 Measured insertion loss with (a) two resonators (b) two resonators and a rejection stub

band pass response. The initial low pass filter has been implemented by cascading two low impedance sections [8]. Two slots have been added in each of these low impedance sections to enhance the ripple amplitude (cf. Fig. 7 (a)). A ripple has been measured to $10 \mathrm{~dB}$ in amplitude as shown in Fig. 8 (a). Then, open stub creating a transmission zero at 36 $\mathrm{GHz}$ has been added to enhance the rejection up to $-35 \mathrm{~dB}$ in the lower band (Fig. 7 (b) and Fig. 8 (b)). Capacitive feeding could be used to eliminate the low frequency pass band if it is required. This combination results in a pass-band response at $60 \mathrm{GHz}$ center frequency and a relative $3-\mathrm{dB}$ bandwidth of $15 \%$. Insertion loss $<15 \mathrm{~dB}$ has been measured. A ripple of $+/-0.15 \mathrm{~dB}$ has been measured over a bandwidth of $6 \mathrm{GHz}$ centered at $60 \mathrm{GHz}$. (Fig 8). The design offers a very simple, low loss and low cost filtering solution for wideband millimeter-wave applications such as $60 \mathrm{GHz}$ WLAN gigabits wireless applications.

\section{Conclusions}

We have presented the development of highly integrated millimeter-wave SOP passive functions enabling a complete passive solution for compact, low cost wireless front-end systems in both multilayer LTCC and LCP technology. The LTCC patch resonator filter that uses vertical coupling overlap and transverse cuts as design parameters achieves a high level of miniaturization and a great compromise between size and power handling. Excellent performance is verified for two operating frequencies $(58-60 \mathrm{GHz} / 38-40 \mathrm{GHz})$ of mobile communication systems through a measured insertion loss $(<$ $2.3 \mathrm{~dB} / 2.3 \mathrm{~dB})$ and a return loss $(>25.3 \mathrm{~dB} / 18.2 \mathrm{~dB})$ over the pass-band, and a bandwidth $(1.67 \% / 3 \%)$ centered at $(59.3$ $\mathrm{GHz} / 39 \mathrm{GHz}$ ). Also, a band filter exploiting the ripple of modified stepped impedance Tchevyscheff low pass filter combined with a rejection stub has been designed to enable a low cost, very easy to design filtering solution to be integrated. Insertion loss as low as $1.5 \mathrm{~dB}$ has been measured demonstrating the potential of LCP for millimeter-wave applications. A ripple of $+/-0.15 \mathrm{~dB}$ has been measured over a bandwidth of $6 \mathrm{GHz}$ centered at $60 \mathrm{GHz}$. The design offers a very simple, low loss and cost-saving filtering solution, ready to be integrated in wideband millimeter-wave applications such as $60 \mathrm{GHz}$ WLAN gigabits wireless systems. In the next step, we will demonstrate additional compact and easy-todesign passive functions, such as filters and antennas with excellent performance and high integration potential. The 3D embedded functions will include patch resonator filters and integrated antennas to provide easy and compact solutions for different applications like mixing and multiplexing. A thorough comparison between LTCC and LCP will focus on the advantages and disadvantages of these two technologies for 3D modules up to $100 \mathrm{GHz}$.

\section{Acknowledgments}

The authors wish to acknowledge the support of the Georgia Tech., Packaging Research Center, the Georgia Electronic Design Center, the NSF Career Award \#ECS9984761, and the NSF Grant \#ECS-0313951.

\section{References}

1. K.Lim et al., "RF-System-On-Package (SOP) for Wireless Communications," IEEE Microwave Magazine, Vol.3, No.1, pp.88-99, March 2002.

2. S. Pinel, M.Davis, V.Sundaram, K.Lim, J.Laskar, G.White and R.Tummala, "High Q passives on Liquid Crystal Polymer substrates and BGA technology for 3D integrated RF Front-End Module", IEICE Transactions on 
Electronics. Vol.E86-C, No.8, pp 1584-1592, August 2003.

3. M.F. Davis, A. Sutono, A. Obatoyinbo, S. Chakraborty, K. Lim, S. Pinel, J. Laskar, S. Lee, R. Tummala, "Integrated RF Function Architectures in Fully-Organic SOP Technology", EPEP2001 - IEEE Electrical Performances of Electronic Packaging Conference, pp 93-96, Cambridge, Massachusetts, Oct. 2001.

4. Kellee Brownlee, Swapan Bhattacharya, Ken-ichi Shinotani, CP Wong and Rao Tummala, "Liquid Crystal Polymer for High Performance SOP Applications", 8th International Symposium on Advanced Packaging Materials, pp 249-253, IEEE 2002.

5. V.Kondratyev et al., "On the design of LTCC filter for millimeter-waves," IEEE MTT-S Int .Microwave Sym. Dig., pp. 1771-1773, Philadelphia, PA, June 2003.

6. David M. Pozar et al., Microstrip Antennas, IEEE press (New York, 1995).

7. Robert E. Collin, Foundations for Microwave Engineering, McGraw Hill (New York, 1992).

8. G.L.Matthaei et al., Microwave filters, impedance-matching networks, and coupling structures, Bookmart Press, (Nort Bergen, 1985). 